\title{
CO-COATOMICALLY SUPPLEMENTED MODULES
}

\author{
R. Alizade ${ }^{1}$ and S. Güngör ${ }^{2}$
}

UDC 512.5

\begin{abstract}
It is shown that if a submodule $N$ of $M$ is co-coatomically supplemented and $M / N$ has no maximal submodule, then $M$ is a co-coatomically supplemented module. If a module $M$ is co-coatomically supplemented, then every finitely $M$-generated module is a co-coatomically supplemented module. Every left $R$-module is co-coatomically supplemented if and only if the ring $R$ is left perfect. Over a discrete valuation ring, a module $M$ is co-coatomically supplemented if and only if the basic submodule of $M$ is coatomic. Over a nonlocal Dedekind domain, if the torsion part $T(M)$ of a reduced module $M$ has a weak supplement in $M$, then $M$ is co-coatomically supplemented if and only if $M / T(M)$ is divisible and $T_{P}(M)$ is bounded for each maximal ideal $P$. Over a nonlocal Dedekind domain, if a reduced module $M$ is co-coatomically amply supplemented, then $M / T(M)$ is divisible and $T_{P}(M)$ is bounded for each maximal ideal $P$. Conversely, if $M / T(M)$ is divisible and $T_{P}(M)$ is bounded for each maximal ideal $P$, then $M$ is a co-coatomically supplemented module.
\end{abstract}

\section{Introduction}

Throughout the paper, $R$ denotes an associative ring with identity and all modules are left unitary $R$-modules $\left({ }_{R} M\right)$, unless otherwise stated. Let $U$ be a submodule of $M$. A submodule $V$ of $M$ is called a supplement of $U$ in $M$ if $V$ is a minimal element in the set of submodules $L \leq M$ with $U+L=M$. The submodule $V$ is a supplement of $U$ in $M$ if and only if $U+V=M$ and $U \cap V \ll V$. A module $M$ is called supplemented if every submodule of $M$ has a supplement in $M$ (see [9], Section 41, or [5], Chapter 4). Semisimple, artinian, and hollow (in particular local) modules are supplemented. A module $M$ is called coatomic if every proper submodule of $M$ is contained in a maximal submodule (see [12]).

Let $N$ be a submodule of a module $M$. We say that $N$ is a co-coatomic submodule in $M$ if $M / N$ is coatomic. Semisimple, finitely generated, and local modules are coatomic modules. Since every factor module of a coatomic module is coatomic, every submodule of semisimple finitely generated and local modules is cocoatomic. A module $M$ is said to be a co-coatomically supplemented module if every co-coatomic submodule of $M$ has a supplement in $M$. A submodule $N$ of $M$ is called cofinite if $M / N$ is finitely generated. $M$ is called a cofinitely supplemented module if every cofinite submodule of $M$ has a supplement in $M$ (see [1]). Clearly, a co-coatomically supplemented module is cofinitely supplemented and a coatomic module is co-coatomically supplemented if and only if it is a supplemented module. A module $M$ is called co-coatomically weak supplemented if every co-coatomic submodule $N$ of $M$ has a weak supplement in $M$, i.e., $N+K=M$ and $N \cap K \ll M$ for some submodule $K$ of $M$. It is clear that a co-coatomically supplemented module is co-coatomically weak supplemented. A submodule $U$ of an $R$-module $M$ has ample supplements in $M$ if, for every submodule $V$ of $M$ with $U+V=M$, there exists a supplement $V^{\prime}$ of $U$ with $V^{\prime} \leq V$ (see [5, p. 237]). A module $M$ is called co-coatomically amply supplemented if every co-coatomic submodule of $M$ has ample supplements in $M$. Clearly, a co-coatomically amply supplemented module is co-coatomically supplemented.

\footnotetext{
${ }^{1}$ Yaşar University, Izmir, Turkey.

${ }^{2}$ Izmir Institute of Technology, Izmir, Turkey.
}

Published in Ukrains'kyi Matematychnyi Zhurnal, Vol.69, No.7, pp.867-876, July, 2017. Original article submitted April 30, 2012, revision submitted April 3, 2017. 
In Section 2, we show that if a submodule $N$ of $M$ is co-coatomically supplemented and $M / N$ has no maximal submodule, then $M$ is co-coatomically supplemented. Every left $R$-module is co-coatomically supplemented if and only if the ring $R$ is left perfect.

In Section 3, we study co-coatomically supplemented modules over a discrete valuation ring. It is shown that a module $M$ is co-coatomically supplemented if and only if the basic submodule of $M$ is coatomic if and only if $M=T(M) \oplus X$, where the reduced part of $T(M)$ is bounded and $X / \operatorname{Rad}(X)$ is finitely generated.

In Section 4, we study co-coatomically supplemented modules over nonlocal Dedekind domains. A torsion module $M$ is co-coatomically weak supplemented if and only if it is co-coatomically supplemented. We show that, for a reduced module $M$, if the torsion part $T(M)$ of $M$ has a weak supplement in $M$, then $M$ is co-coatomically supplemented if and only if $M / T(M)$ is divisible and $T_{P}(M)$ is bounded for each maximal ideal $P$. For a reduced module $M$, if $M$ is co-coatomically amply supplemented, then $M / T(M)$ is divisible and $T_{P}(M)$ is bounded for each maximal ideal $P$ of $R$. Conversely, if $M / T(M)$ is divisible and $T_{P}(M)$ is bounded for each maximal ideal $P$ of $R$, then $M$ is a co-coatomically supplemented module.

\section{Co-Coatomically Supplemented Modules}

For any module $M, \operatorname{Soc}(M)$ denotes the socle of $M$ and $\operatorname{Rad}(M)$ denotes the radical of $M$. The Jacobson radical of ${ }_{R} R$ is denoted by $\operatorname{Jac}(R)$.

Let $\left\{M_{\lambda}\right\}_{\lambda \in \Lambda}$ be the family of simple submodules of $M$ that are direct summands of $M$. By $\operatorname{Soc}^{\oplus}(M)$ we denote the sum of $M_{\lambda}$ s for all $\lambda \in \Lambda$, i.e.,

$$
\operatorname{Soc}^{\oplus}(M)=\sum_{\lambda \in \Lambda} M_{\lambda}
$$

Clearly,

$$
\operatorname{Soc}^{\oplus}(M) \leq \operatorname{Soc}(M)
$$

Theorem 2.1. Let $R$ be a ring. The following assertions are equivalent for an $R$-module $M$ :

1. Every co-coatomic submodule of $M$ is a direct summand of $M$.

2. Every cofinite submodule of $M$ is a direct summand of $M$.

3. Every maximal submodule of $M$ is a direct summand of $M$.

4. $M / \operatorname{Soc}^{\oplus}(M)$ does not contain a maximal submodule.

5. $M / \operatorname{Soc}(M)$ does not contain a maximal submodule.

Proof. (1) $\Rightarrow(2)$ is clear since every cofinite submodule is co-coatomic.

(2) $\Rightarrow$ (3). Clear.

(3) $\Rightarrow$ (4). Suppose that $M / \operatorname{Soc}^{\oplus}(M)$ contains a maximal submodule $K / \operatorname{Soc}^{\oplus}(M)$. Thus, $K$ is a maximal submodule of $M$. By the hypothesis, $M=K \oplus K^{\prime}$ and $K^{\prime}$ is simple. Hence, we get

$$
K^{\prime} \leq \operatorname{Soc}^{\oplus}(M) \leq K
$$

A contradiction. 
(4) $\Rightarrow(5)$. This is clear because $\operatorname{Soc}^{\oplus}(M) \leq \operatorname{Soc}(M)$.

(5) $\Rightarrow(1)$. Let $N$ be a co-coatomic submodule of $M$. Since

$$
M /(N+\operatorname{Soc}(M)) \cong(M / N) /((N+\operatorname{Soc}(M)) / N)
$$

and $M / N$ is coatomic, we conclude that $M /(N+\operatorname{Soc}(M))$ is also coatomic. Since $M / \operatorname{Soc}(M)$ has no maximal submodule, $M /(N+\operatorname{Soc}(M))$ also has no maximal submodule. Therefore, $M=N+\operatorname{Soc}(M)$. It follows that $M=N \oplus N^{\prime}$ for any submodule $N^{\prime}$ such that

$$
\operatorname{Soc}(M)=(N \cap \operatorname{Soc}(M)) \oplus N^{\prime}
$$

A supplemented module is co-coatomically supplemented but co-coatomically supplemented modules need not be supplemented as shown in the following example:

Example 2.1. The $\mathbb{Z}$-module $\mathbb{Q}$ is co-coatomically supplemented since the only co-coatomic submodule is $\mathbb{Q}$ itself. At the same time, the $\mathbb{Z}$-module $\mathbb{Q}$ is not supplemented because $\mathbb{Q}$ is not torsion (see [10], Theorem 3.1).

Proposition 2.1. Let $M$ be a semilocal module with small radical $\operatorname{Rad}(M)$. Then $M$ is co-coatomically supplemented if and only if $M$ is supplemented.

Proof. Let $N$ be a submodule of $M$. Since $M$ is semilocal, $M / \operatorname{Rad}(M)$ is semisimple, i.e., coatomic. Consider the following statement:

$$
M /(N+\operatorname{Rad}(M)) \cong(M / \operatorname{Rad}(M)) /((N+\operatorname{Rad}(M)) / \operatorname{Rad}(M))
$$

Since $M / \operatorname{Rad}(M)$ is coatomic, $M /(N+\operatorname{Rad}(M))$ is also coatomic. Therefore, $N+\operatorname{Rad}(M)$ has a supplement in $M$, say, $K$. Then

$$
M=N+\operatorname{Rad}(M)+K \quad \text { and } \quad(N+\operatorname{Rad}(M)) \cap K \ll K
$$

Since $\operatorname{Rad}(M) \ll M$, we conclude that $M=N+K$ and

$$
N \cap K \leq(N+\operatorname{Rad}(M)) \cap K \ll K
$$

Thus, $M$ is supplemented.

A co-coatomically supplemented module is cofinitely supplemented but the example presented in what follows shows that a cofinitely supplemented module is not necessarily co-coatomically supplemented.

A ring $R$ is called semiperfect if $R / \operatorname{Jac}(R)$ is semisimple and the idempotents in $R / \operatorname{Jac}(R)$ can be lifted to $R$ (see [9], 42.6).

A ring is called left perfect if $R / \operatorname{Jac}(R)$ is left semisimple and $\operatorname{Jac}(R)$ is right t-nilpotent (see [9], 43.9).

By ${ }_{R} R^{(\mathbb{N})}$ we denote the direct sum of $R$-module $R$ by the index set $\mathbb{N}$. Note that $\mathbb{N}$ denotes the set of all positive integers.

Any direct sum of cofinitely supplemented modules is cofinitely supplemented [1] (Corollary 2.4).

Example 2.2. Let $p$ be a prime integer. We consider the following ring:

$$
R=\mathbb{Z}_{(p)}=\left\{\frac{a}{b} \mid a, b \in \mathbb{Z}, b \neq 0,(b, p)=1\right\},
$$


which is the localization of $\mathbb{Z}$ at $(p)$. In this case, the $R$-module $R$ is supplemented. Then the $R$-module $R^{(\mathbb{N})}$ is cofinitely supplemented by [1] (Corollary 2.4). Furthermore, $R$ is a semiperfect ring and, therefore, $R / \operatorname{Jac}(R)$ is semisimple (see [9], 42.6). Hence, $R$ is semilocal. However, $R$ is not a perfect ring because its Jacobson radical is not $t$-nilpotent by [9] (43.9). Note that $\operatorname{Rad}\left({ }_{R} R^{(\mathbb{N})}\right)$ is a co-coatomic submodule of ${ }_{R} R^{(\mathbb{N})}$ but $\operatorname{Rad}\left({ }_{R} R^{(\mathbb{N})}\right)$ does not have a supplement in ${ }_{R} R^{(\mathbb{N})}$ because $R$ is not a perfect ring (see [3], Theorem 1). Hence, $R_{R}^{(\mathbb{N})}$ is not co-coatomically supplemented.

Example 2.2 shows that the cofinitely supplemented modules and co-coatomically supplemented modules not necessarily coincide over semiperfect rings and discrete valuation rings.

Proposition 2.2. A factor module of a co-coatomically supplemented module is co-coatomically supplemented.

Proof. Let $M$ be a co-coatomically supplemented module and let $N$ be a submodule of $M$. Then any cocoatomic submodule of $M / N$ is a submodule of the form $L / N$, where $L$ is co-coatomic submodule of $M$. By the hypothesis, $L$ has a supplement in $M$, say, $K$. This implies that $(K+N) / N$ is a supplement of $L / N$ in $M / N$ by [9] (41.1(7)).

Proposition 2.3. Let $M$ be a co-coatomically supplemented module. Then every co-coatomic submodule of the module $M / \operatorname{Rad}(M)$ is a direct summand.

Proof. Any co-coatomic submodule of $M / \operatorname{Rad}(M)$ has the form $N / \operatorname{Rad}(M)$, where $N$ is a co-coatomic submodule of $M$. Since $M$ is co-coatomically supplemented, there exists a submodule $K$ of $M$ such that $M=$ $N+K$ and $N \cap K \ll K$. This yields $N \cap K \leq \operatorname{Rad}(M)$. Thus,

$$
\begin{gathered}
M / \operatorname{Rad}(M)=(N / \operatorname{Rad}(M))+((K+\operatorname{Rad}(M)) / \operatorname{Rad}(M)), \\
(N / \operatorname{Rad}(M)) \cap((K+\operatorname{Rad}(M)) / \operatorname{Rad}(M))=(N \cap K+\operatorname{Rad}(M)) / \operatorname{Rad}(M)=0 .
\end{gathered}
$$

Hence,

$$
M / \operatorname{Rad}(M)=(N / \operatorname{Rad}(M)) \oplus((K+\operatorname{Rad}(M)) / \operatorname{Rad}(M)) .
$$

To prove that a finite sum of co-coatomically supplemented modules is a co-coatomically supplemented module, we use the following standard lemma (see [9], 41.2):

Lemma 2.1. Let $N$ and $L$ be submodules of an $R$-module $M$ such that $N$ is co-coatomic, $L$ is cocoatomically supplemented, and $N+L$ has a supplement in $M$. Then $N$ has a supplement in $M$.

Proof. Let $K$ be a supplement of $N+L$ in $M$. Note that

$$
L /(L \cap(N+K)) \cong(N+K+L) /(N+K)=M /(N+K) .
$$

This module is coatomic and, therefore, there is a supplement $H$ of $L \cap(N+K)$ in $L$, i.e.,

$$
L=H+L \cap(N+K) \quad \text { and } \quad H \cap L \cap(N+K) \ll H .
$$

Hence,

$$
M=N+L+K=N+K+H+L \cap(N+K)=N+K+H,
$$




$$
\begin{aligned}
N \cap(H+K) & \leq H \cap(N+K)+K \cap(N+H) \\
& \leq H \cap(N+K)+K \cap(N+L) \ll H+K .
\end{aligned}
$$

Therefore, $H+K$ is a supplement of $N$ in $M$.

A (direct) sum of infinitely many co-coatomically supplemented modules need not be co-coatomically supplemented by Example 2.2 but a finite sum of co-coatomically supplemented modules is always co-coatomically supplemented.

Theorem 2.2. A finite sum of co-coatomically supplemented modules is co-coatomically supplemented.

Proof. Clearly, it is sufficient to prove that the sum $M=M_{1}+M_{2}$ of two co-coatomically supplemented modules $M_{1}$ and $M_{2}$ is a co-coatomically supplemented. Let $U$ be a co-coatomic submodule of $M$. Then $M=$ $M_{1}+M_{2}+U$. Since $M_{2}+U$ is a co-coatomic submodule of $M$ and $M_{1}$ is co-coatomically supplemented, $M_{2}+U$ has a supplement in $M$ by Lemma 2.1. Since $M_{2}$ is co-coatomically supplemented and $U$ is co-coatomic, by Lemma 2.1, $U$ has a supplement in $M$. Thus, $M$ is co-coatomically supplemented.

Let $M$ and $N$ be $R$-modules. If there is an epimorphism $f: M^{(\Lambda)} \rightarrow N$ for some finite set $\Lambda$, then $N$ is called a finitely $M$-generated module.

The following assertion is a corollary of Proposition 2.2 and Theorem 2.2:

Corollary 2.1. If $M$ is co-coatomically supplemented module, then any finitely $M$-generated module is a cocoatomically supplemented module.

A ring $R$ is called a left $V$-ring if every simple $R$-module is injective (see [9, p. 192]). A commutative ring $R$ is a $V$-ring if and only if $R$ is a von Neumann regular ring (see [9], 23.5).

Proposition 2.4. A module $M$ over a $V$-ring $R$ is co-coatomically supplemented if and only if $M$ is semisimple.

Proof. $(\Leftarrow)$ Clear.

$(\Rightarrow)$ Since $M$ is a co-coatomically supplemented module, $M / \operatorname{Soc}(M)$ has no maximal submodule by Theorem 2.1. It follows from [9] (23.1) that

$$
M / \operatorname{Soc}(M)=\operatorname{Rad}(M / \operatorname{Soc}(M))=0
$$

because $R$ is a $V$-ring. Thus, $M$ is semisimple.

Corollary 2.2. Any direct sum of co-coatomically supplemented modules is co-coatomically supplemented over a left $V$-ring.

Proof. By Proposition 2.4, co-coatomically supplemented and semisimple modules coincide over left $V$-rings.

Theorem 2.3. Let $N$ be a co-coatomically supplemented submodule of an $R$-module $M$ such that $M / N$ has no maximal submodule. Then $M$ is a co-coatomically supplemented module.

Proof. Let $L$ be a submodule of $M$ such that $M / L$ is coatomic. Clearly, $M /(N+L)$ is also coatomic. Since $M / N$ has no maximal submodule, $M /(N+L)$ also has no maximal submodule. Therefore, $M=N+L$. By Lemma 2.1, $L$ has a supplement in $M$. Thus, $M$ is a co-coatomically supplemented module. 
The following corollary is a direct result of Theorem 2.3:

Corollary 2.3. Let $M$ be a module and let $M / \operatorname{Soc}(M)$ have no maximal submodule. Then $M$ is cocoatomically supplemented.

Proposition 2.5. Let $M$ be a co-coatomically supplemented $R$-module. If $M$ contains a maximal submodule, then $M$ contains a local submodule.

Proof. Let $L$ be a maximal submodule of $M$. Then $L$ is a co-coatomic submodule of $M$. Since $M$ is a cocoatomically supplemented module, there exists a submodule $K$ of $M$ such that $K$ is a supplement of $L$ in $M$, i.e., $M=K+L$ and $K \cap L \ll K$. It follows from [9] (41.1(3)) that $K$ is local.

A module $M$ is called linearly compact if, for any family of cosets $\left\{x_{i}+M_{i}\right\} \triangle, x_{i} \in M$, and submodules $M_{i} \leq M$ (with finitely cogenerated $M / M_{i}$ ), the intersection of any group of finitely many cosets from this family is nonempty, then the intersection of the entire family of cosets is also nonempty (see [9], 29.7(c)).

The following proposition gives a characterization of a co-coatomically supplemented module by a linearly compact submodule:

Proposition 2.6. Let $K$ be a linearly compact submodule of an $R$-module $M$. Then $M$ is co-coatomically supplemented if and only if $M / K$ is co-coatomically supplemented.

Proof. $(\Rightarrow)$ By Proposition 2.2.

$(\Leftarrow)$ Let $N$ be a co-coatomic submodule of $M$. Then $(N+K) / K$ is co-coatomic submodule of $M / K$ because $N+K$ is co-coatomic submodule of $M$. Since $M / K$ is co-coatomically supplemented, $(N+K) / K$ has a supplement in $M / K$. The submodule $K$ has a supplement in every submodule $L$ of $M$ with $K \leq L$ because $K$ is linearly compact (see [8], Lemma 2.3). Moreover, $K$ is supplemented by [9] (29.8(2)) and [8] (Lemma 2.3). Therefore, $N$ has a supplement in $M$ by [8] (Corollary 2.7). Thus, $M$ is co-coatomically supplemented.

Remark 2.1. A module $M$ is called $\Sigma$-selfprojective if, for each index set $I$, the module $M^{(I)}$ is selfprojective. For an $R$-module $M$, if $M$ is $\Sigma$-selfprojective and $U \leq \operatorname{Rad}(M)$, then the following assertion is true: $U$ has a supplement in $M$ and, hence, $U$ is small in $M$ [11] (Satz 4.1). Clearly, ${ }_{R} R^{(\mathbb{N})}$ is $\Sigma$-selfprojective and

$$
\operatorname{Rad}\left({ }_{R} R^{(\mathbb{N})}\right) \leq \operatorname{Rad}\left({ }_{R} R^{(\mathbb{N})}\right)
$$

Therefore, if $\operatorname{Rad}\left({ }_{R} R^{(\mathbb{N})}\right)$ has a supplement in ${ }_{R} R^{(\mathbb{N})}$, then

$$
\operatorname{Rad}\left({ }_{R} R^{(\mathbb{N})}\right) \ll{ }_{R} R^{(\mathbb{N})} .
$$

Theorem 2.4. Every left $R$-module is co-coatomically supplemented if and only if the ring $R$ is left perfect.

Proof. $(\Leftarrow)$ Clear.

$(\Rightarrow)$ By the hypothesis, every left $R$-module is co-coatomically supplemented and, hence, every left $R$-module is cofinitely supplemented. Then $R$ is semiperfect by [1] (Theorem 2.13). Thus, $R / \mathrm{Jac}(R)$ is semisimple by [9] (42.6). This means that ${ }_{R} R^{(\mathbb{N})} / \operatorname{Rad}\left({ }_{R} R^{(\mathbb{N})}\right)$ is semisimple. Therefore, $\operatorname{Rad}\left({ }_{R} R^{(\mathbb{N})}\right)$ is co-coatomic in ${ }_{R} R^{(\mathbb{N})}$. By the hypothesis, $\operatorname{Rad}\left({ }_{R} R^{(\mathbb{N})}\right)$ has a supplement in ${ }_{R} R^{(\mathbb{N})}$. By Remark 2.1,

$$
\operatorname{Rad}\left({ }_{R} R^{(\mathbb{N})}\right) \ll{ }_{R} R^{(\mathbb{N})} .
$$

Since $R / \operatorname{Jac}(R)$ is semisimple and $\operatorname{Rad}\left({ }_{R} R^{(\mathbb{N})}\right) \ll{ }_{R} R^{(\mathbb{N})},{ }_{R} R$ is perfect by [9] (43.9). Thus, the ring $R$ is left perfect. 


\section{Co-Coatomically Supplemented Modules Over Discrete Valuation Rings}

Throughout this section $R$ is a discrete valuation ring. An $R$-module $M$ is called radical-supplemented if $\operatorname{Rad}(M)$ has a supplement in $M$ (see [11]). A module $M$ is radical supplemented if and only if the basic submodule of $M$ is coatomic (see [11], Satz 3.1). A module $M$ is coatomic if and only if $M$ is reduced and supplemented (see [10], Lemma 2.1).

Proposition 3.1. Let $M$ be an R-module. Then $M$ is a co-coatomically supplemented module if and only if the basic submodule of $M$ is coatomic.

Proof. $(\Rightarrow) M / \operatorname{Rad}(M)=M / p M$ is semisimple and, therefore, coatomic. Since $M$ is a co-coatomically supplemented module, $p M$ has a supplement. Thus, $M$ is a radical-supplemented module. Then the basic submodule of $M$ is coatomic by [11] (Satz 3.1).

$(\Leftarrow)$ Let $X$ be a submodule of $M$ such that $M / X$ is coatomic and let $B$ be the basic submodule of $M$. Then $M /(X+B)$ is also coatomic. Furthermore, $M /(X+B)$ is reduced by [10] (Lemma 2.1). On the other hand, $M /(X+B)$ is divisible because $M / B$ is divisible. Therefore, $M /(X+B)=0$, i.e., $M=X+B$. By the hypothesis, $B$ is coatomic and, hence, supplemented by [10] (Lemma 2.1). Therefore, $X$ has a supplement in $M$ by Lemma 2.1 . Hence, $M$ is a co-coatomically supplemented module.

Corollary 3.1. Co-coatomically supplemented modules and radical supplemented modules coincide.

The following corollary is a consequence of [11] (Satz 3.1) and Corollary 3.1:

Corollary 3.2. A module $M$ is co-coatomically supplemented if and only if $M=T(M) \oplus X$, where the reduced part of $T(M)$ is bounded and $X / \operatorname{Rad}(X)$ is finitely generated.

The following properties were presented in [11] (Lemma 3.2) for the radical-supplemented modules over a discrete valuation ring. Since co-coatomically supplemented modules coincide with radical-supplemented modules, these properties clearly hold for the co-coatomically supplemented modules:

Corollary 3.3. For an R-module $M$ the following assertions are true:

1. The class of co-coatomically supplemented modules is closed under pure submodules and extensions.

2. If $M$ is co-coatomically supplemented and $M / U$ is reduced, then $U$ is also co-coatomically supplemented.

3. Every submodule of $M$ is co-coatomically supplemented if and only if $T(M)$ is supplemented and $M / T(M)$ has a finite rank.

\section{Co-Coatomically Supplemented Modules over Nonlocal Dedekind Domains}

Throughout this section, $R$ is a nonlocal Dedekind domain, unless otherwise stated.

Theorem 4.1. Let $R$ be a Dedekind domain and let $M$ be an R-module. Then $M$ is a module whose cocoatomic submodules are direct summands if and only if

1) $T(M)=M_{1} \oplus M_{2}$, where $M_{1}$ is semisimple and $M_{2}$ is divisible,

2) $M / T(M)$ is divisible. 
Proof. By Theorem 2.1 and [4] (Theorem 6.11).

A submodule $N$ of a module $M$ has (is) a weak supplement in $M$ if $M=N+K$ and $N \cap K \ll M$ for some submodule $K$ of $M$. Clearly, every supplement is a weak supplement.

Recall that, over an arbitrary $\operatorname{ring} R$, a module $M$ is called co-coatomically weak supplemented if every co-coatomic submodule has a weak supplement in $M$.

Proposition 4.1. Over an arbitrary ring, a small cover of a co-coatomically weak supplemented module is co-coatomically weak supplemented.

Proof. Let $M$ be a small cover of a co-coatomically weak supplemented module $N$. Then $N \cong M / K$ for some $K \ll M$. We take a co-coatomic submodule $L$ of $M$. Thus, $(L+K) / K$ is a co-coatomic submodule of $M / K$ because $L+K$ is a co-coatomic submodule of $M$. By the hypothesis, $M / K$ is co-coatomically weak supplemented and, hence, $(L+K) / K$ has a weak supplement in $M / K$, say, $X / K$. Since $K \ll M$, we get

$$
(X \cap L)+K=X \cap(L+K) \ll M
$$

(see [5], 2.2(3)). Therefore,

$$
M=L+X \quad \text { and } \quad L \cap X \ll M,
$$

i.e., $X$ is a weak supplement of $L$ in $M$. Thus, $M$ is co-coatomically weak supplemented.

Proposition 4.2. Over an arbitrary ring, a factor module of a co-coatomically weak supplemented module is co-coatomically weak supplemented.

Proof. Let $M$ be a co-coatomically weak supplemented module and let $N$ be a submodule of $M$. Then any co-coatomic submodule of $M / N$ is a submodule of the form $L / N$, where $L$ is a co-coatomic submodule of $M$. By the hypothesis, $L$ has a weak supplement in $M$, say, $K$. Thus, $(K+N) / N$ is a weak supplement of $L / N$ in $M / N$ by [5] (2.2(5)).

Let $M$ be a module and let $K$ be a submodule of $M$. A submodule $L$ of $M$ is called a complement of $K$ in $M$ if it is maximal in the set of all submodules $N$ of $M$ with $K \cap N=0$. A submodule $L$ of $M$ is called a complement submodule if it is a complement of some submodule of $M$ (see [5], 1.9). A submodule of $M$ is a complement if and only if it is closed (see [5], 1.10). A submodule $L$ of $M$ is called coclosed in $M$ if $L$ has no proper submodules $K$ for which $L / K \ll M / K$ (see [5], 3.6). Over a Dedekind domain, a submodule $N$ of $M$ is closed if and only if $N$ is coclosed (see [10], Lemma 3.3). Over a domain $R$, a torsion submodule $T(M)$ of a module $M$ is a closed submodule of $M$ (see [7], Example 6.34). Therefore, over a Dedekind domain, a torsion submodule $T(M)$ of a module $M$ is a coclosed submodule of $M$.

Proposition 4.3. Let $M$ be a torsion R-module. Then $M$ is co-coatomically weak supplemented if and only if it is co-coatomically supplemented.

Proof. $(\Leftarrow)$ Clear.

$(\Rightarrow)$ Let $K$ be a submodule of $M$ such that $M / K$ is coatomic. Since $M$ is co-coatomically weak supplemented, $K$ has a weak supplement in $M$, say, $N$. Then

$$
M=K+N \quad \text { and } \quad K \cap N \ll M .
$$

Since $M$ is a torsion, $N$ is also a torsion and, hence, it is coclosed. Therefore, $K \cap N \ll N$ by [5] (3.7(3)). Thus, $M$ is co-coatomically supplemented. 
Let $R$ be a Dedekind domain and let $\mathcal{P}$ be the set of all maximal ideals of $R$. For some $P \in \mathcal{P}$, the submodule

$$
\left\{m \in M \mid P^{n} m=0 \text { for some integer } n \geq 1\right\}
$$

is said to be the $P$-primary component of $M$. This submodule is denoted by $T_{P}(M)$.

Over a discrete valuation ring, if a module $M$ is torsion and reduced and the radical of $M$ has a supplement in $M$, then $M$ is bounded (see [10, p. 48], 2nd Folgerung).

Theorem 4.2. Let $M$ be a reduced R-module. If $T(M)$ has a weak supplement in $M$, then $M$ is cocoatomically supplemented if and only if $M / T(M)$ is divisible and $T_{P}(M)$ is bounded for each maximal ideal $P$.

Proof. $\quad(\Rightarrow)$ Let $M$ be a co-coatomically supplemented reduced $R$-module. Then the module $M / T(M)$ is radical: Suppose $K$ is a maximal submodule of $M$ with $T(M) \subseteq K$. Since $M$ is co-coatomically supplemented, $K$ has a supplement, say, $V$. Since $K$ is maximal, $V$ is local and, therefore, $V$ is cyclic, i.e., $V \cong R / I$ (see [9], 41.1(3)). On the other hand, $R$ is nonlocal and, thus, $I \neq 0$, i.e., $V$ is torsion. Hence, $V \subseteq T(M)$; a contradiction. Therefore, $M / T(M)$ has no maximal submodule and, thus, $M / T(M)$ is divisible (see [1], Lemma 4.4). By [7] (Example 6.34), $T(M)$ is closed, i.e., it is coclosed by [10] (Lemma 3.3). Since $T(M)$ has a weak supplement, it is a supplement by [5] (20.2). Hence, there is a submodule $N$ in $M$ such that

$$
T(M)+N=M \quad \text { and } \quad T(M) \cap N \ll T(M) .
$$

Then

$$
T(M) / T(M) \cap N \cong(T(M)+N) / N=M / N
$$

Since $M$ is co-coatomically supplemented, it is co-coatomically weak supplemented and, thus,

$$
T(M) / T(M) \cap N
$$

is co-coatomically weak supplemented. By Proposition 4.1, $T(M)$ is co-coatomically weak supplemented. By Proposition 4.2, $T_{P}(M)$ is also co-coatomically weak supplemented for each $P$ as it is a direct summand of $T(M)$. Moreover, $T_{P}(M)$ is a co-coatomically supplemented module by Proposition 4.3. Thus, $T_{P}(M)$ is bounded for each maximal ideal $P$ (see [10, p. 48], 2nd Folgerung).

$(\Leftarrow)$ Each $T_{P}(M)$ is bounded and, hence, it is supplemented by [10] (Lemma 2.1). Therefore, $T(M)$ is supplemented by [10] (Theorem 3.1). Now let $K$ be a submodule of $M$ such that $M / K$ is coatomic. Then $M /(K+T(M))$ is also coatomic. By the hypothesis, $M / T(M)$ is divisible, i.e., it has no maximal submodules (see [1], Lemma 4.4). Therefore, $M=K+T(M)$. By Lemma 2.1, $K$ has a supplement in $M$. Hence, $M$ is co-coatomically supplemented.

Remark 4.1. We see that the "if" part of the theorem is true without the condition that " $T(M)$ has a weak supplement in $M$." We do not know whether this condition is necessary for the "only if" part.

Corollary 4.1. Let $R$ be a nonlocal Dedekind domain and let $M$ be a reduced $R$-module. If $\operatorname{Rad}(T(M)) \ll$ $T(M)$, then $M$ is co-coatomically supplemented if and only if $M / T(M)$ is divisible.

Proof. $(\Rightarrow)$ Clear by the proof of Theorem 4.2. 
$(\Leftarrow)$ By [2] (Corollary 4.1.2.), $T(M) / \operatorname{Rad}(T(M))$ is semisimple and, thus, it is co-coatomically weak supplemented. Then $T(M)$ is co-coatomically weak supplemented because

$$
\operatorname{Rad}(T(M)) \ll T(M)
$$

by Proposition 4.1. Therefore, $T(M)$ is co-coatomically supplemented by Proposition 4.3. Since $M / T(M)$ is divisible, $M / T(M)$ has no maximal submodule. Hence, $M$ is co-coatomically supplemented by Theorem 2.3.

Theorem 4.3. Let $R$ be a nonlocal Dedekind domain and let $M$ be a reduced $R$-module. If $M$ is cocoatomically amply supplemented, then $M / T(M)$ is divisible and $T_{P}(M)$ is bounded for each $P \in \mathcal{P}$.

Conversely, if $M / T(M)$ is divisible and $T_{P}(M)$ is bounded for each maximal ideal $P$ of $R$, then $M$ is co-coatomically supplemented.

Proof. Let $R$ be a nonlocal Dedekind domain and let $M$ be a co-coatomically amply supplemented reduced $R$-module. Then, by the proof of Theorem 4.2, $M / T(M)$ is divisible. We now suppose that $T_{P}(M)$ is not bounded for some $P \in \mathcal{P}$. If a basic submodule $B_{p}(M)$ is bounded, then, by [6] (Theorem 5), we get

$$
T_{P}(M)=B_{P}(M) \oplus D
$$

where $D$ is divisible. Therefore, $M$ is not reduced and we arrive at a contradiction. Hence, $B_{p}(M)$ is not bounded. We now prove that $B_{P}(M)$ is co-coatomically supplemented. Let $K$ be a co-coatomic submodule of $B_{P}(M)$, i.e., $B_{P}(M) / K$ is coatomic. Thus, $B_{P}(M) / K$ is bounded by [10, p. 48] (2nd Folgerung). We get the following commutative diagram with exact rows and columns:

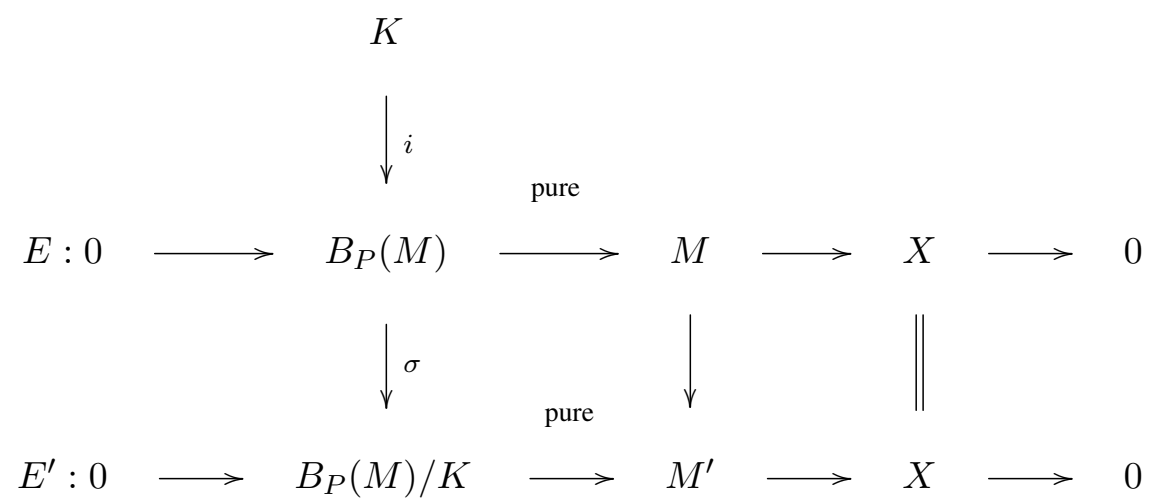

Since $E$ is pure $E^{\prime}$ is also pure. Hence, $E^{\prime}$ is splitting because $B_{P}(M) / K$ is bounded (see [6], Theorem 5). By applying Ext, we obtain the exact sequence

$$
\rightarrow \operatorname{Ext}_{R}(X, K) \stackrel{i_{*}}{\rightarrow} \operatorname{Ext}_{R}\left(X, B_{P}(M)\right) \stackrel{\sigma_{*}}{\rightarrow} \operatorname{Ext}_{R}\left(X, B_{P}(M) / K\right) \rightarrow .
$$

Since

$$
\operatorname{Ext}\left(X, B_{P}(M) / K\right)=0
$$

we find $\sigma_{*}(E)=0$ and, therefore, $E \in \operatorname{Ker} \sigma_{*}=\operatorname{Im} i_{*}$. Thus, there exists a short exact sequence

$$
E^{\prime \prime}: 0 \rightarrow K \rightarrow N \rightarrow X \rightarrow 0
$$


such that $i_{*}\left(E^{\prime \prime}\right)=E$. Hence, we arrive at the following diagram:

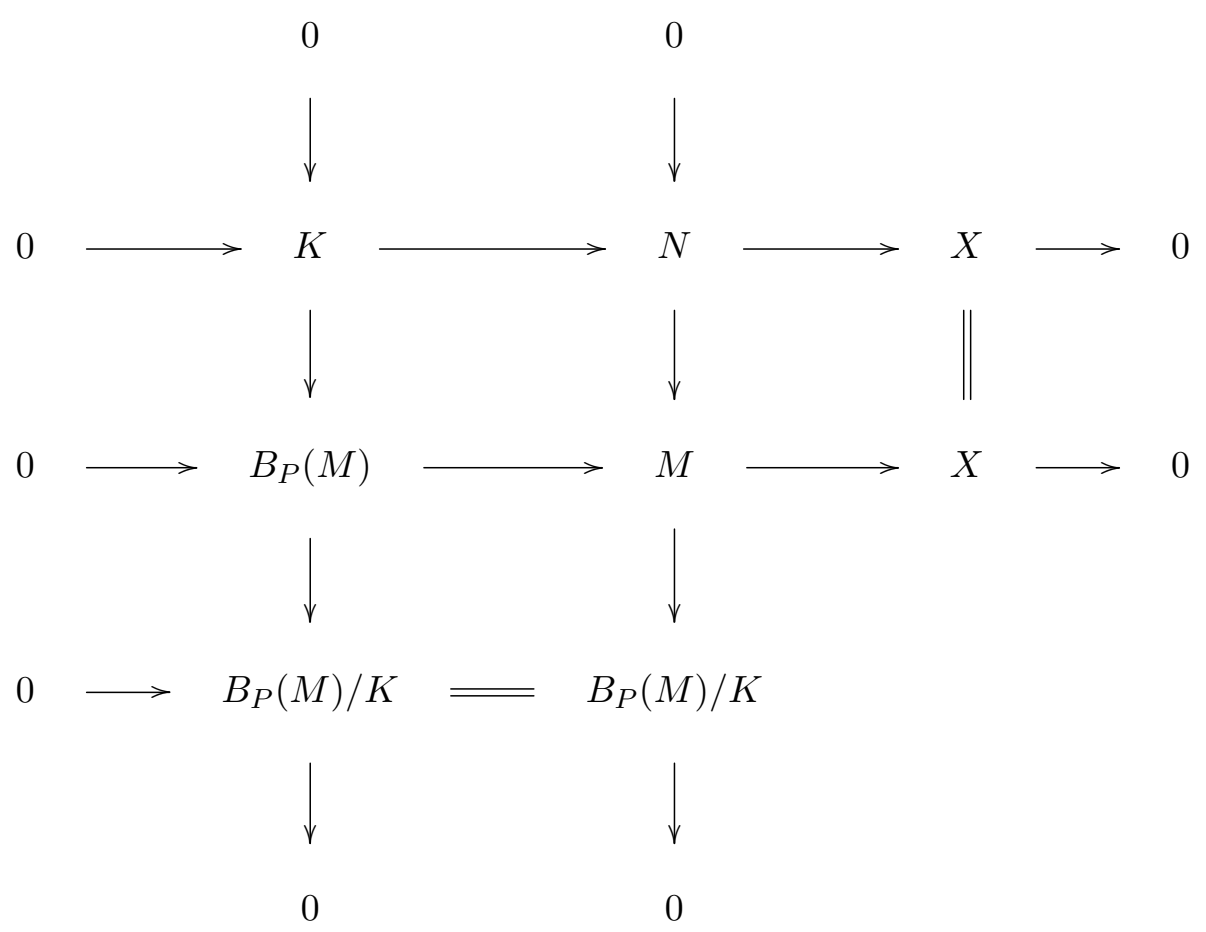

Without loss of generality, we can assume that $K, B_{P}(M)$, and $N$ are submodules of $M$. In this diagram,

$$
B_{P}(M) \cap N=K \quad \text { and } \quad B_{P}(M)+N=M
$$

(see [9]; the Noether isomorphism theorem). Moreover, $M / N$ is coatomic. Since $M$ is co-coatomically amply supplemented, there exists a submodule $L$ of $B_{P}(M)$ such that

$$
N+L=M \quad \text { and } \quad N \cap L \ll L
$$

Therefore,

$$
B_{P}(M)=B_{P}(M) \cap(N+L)=L+\left(B_{P}(M) \cap N\right)=L+K
$$

and

$$
L \cap K \leq L \cap N \ll L .
$$

Thus, $K$ has a supplement in $B_{P}(M)$ and, hence, $B_{P}(M)$ is co-coatomically supplemented. Therefore, $B_{P}(M)$ is bounded by [10, p. 48] (2ndFolgerung). This is a contradiction. This means that $T_{P}(M)$ is bounded for each $P \in \mathcal{P}$.

The converse assertion is clear by Theorem 4.2.

\section{Acknowledgements}

The authors would like to express their deep gratitude to E. Büyükaşık for his support during the preparation of the present paper. 


\section{REFERENCES}

1. R. Aizade, G. Bilhan, and P. F. Smith, "Modules whose maximal submodules have supplements," Comm. Algebra, 29, 2389-2405 (2001).

2. E. Büyükaşık, Weakly and Cofinitely Weak Supplemented Modules over Dedekind Ring: PhD Thesis, Dokuz Eylül University (2005).

3. E. Büyükaşık and C. Lomp, "Rings whose modules are weakly supplemented are perfect. Applications to certain ring extensions," Math. Scand., 105, 25-30 (2009).

4. E. Büyükaşık and D. Pusat-Yılmaz, "Modules whose maximal submodules are supplements," Hacet. J. Math. Stat., 39, No. 4, 477-487 (2010).

5. J. Clark, C. Lomp, N. Vanaja, and R. Wisbauer, Lifting Modules, Birkhäuser-Verlag (2006).

6. I. Kaplansky, “Modules over Dedekind rings and valuation rings," Trans. Amer. Math. Soc., 72, 327-340 (1952).

7. T. Y. Lam, Lectures on Modules and Rings, Springer (1999).

8. P. F. Smith, "Finitely generated supplemented modules are amply supplemented," Arab. J. Sci. Eng., 25, 69-80 (2000).

9. R. Wisbauer, Foundations of Modules and Rings, Gordon \& Breach (1991).

10. H. Zöschinger, "Komplementierte moduln über Dedekindringen,” J. Algebra, 29, 42-56 (1974).

11. H. Zöschinger, "Moduln die in jeder Erweiterung ein Komplement haben," Math. Scand., 35, $267-287$ (1974).

12. H. Zöschinger and F. Rosenberg, "Koatomare moduln,” Math. Z., 170, 221-232 (1980). 